\title{
Borreliacidal activity of Borrelia metal transporter A (BmtA) binding small molecules by manganese transport inhibition
}

This article was published in the following Dove Press journal:

Drug Design, Development and Therapy

II February 2015

Number of times this article has been viewed

\author{
Dhananjay Wagh* \\ Venkata Raveendra \\ Pothineni* \\ Mohammed Inayathullah \\ Song Liu \\ Kwang-Min Kim \\ Jayakumar Rajadas
}

Biomaterials and Advanced Drug

Delivery Laboratory, Stanford

Cardiovascular Pharmacology

Division, Cardiovascular Institute,

Stanford University School of

Medicine, Palo Alto, CA, USA

*These authors contributed equally to this work
Correspondence: Jayakumar Rajadas School of Medicine, Stanford university, 1050 Arastradero Road, Building A, Room AI48, Palo Alto, CA 94304, USA

Tel +l 6507246806

Fax +l 6507244694

Email jayraja@stanford.edu
Abstract: Borrelia burgdorferi, the causative agent of Lyme disease, utilizes manganese (Mn) for its various metabolic needs. We hypothesized that blocking Mn transporter could be a possible approach to inhibit metabolic activity of this pathogen and eliminate the infection. We used a combination of in silico protein structure prediction together with molecular docking to target the Borrelia metal transporter A (BmtA), a single known Mn transporter in Borrelia and screened libraries of FDA approved compounds that could potentially bind to the predicted BmtA structure with high affinity. Tricyclic antihistamines such as loratadine, desloratadine, and 3-hydroxydesloratadine as well as yohimbine and tadalafil demonstrated a tight binding to the in silico folded BmtA transporter. We, then, tested borreliacidal activity and dose response of the shortlisted compounds from this screen using a series of in vitro assays. Amongst the probed compounds, desloratadine exhibited potent borreliacidal activity in vitro at and above $78 \mu \mathrm{g} / \mathrm{mL}(250 \mu \mathrm{M})$. Borrelia treated with lethal doses of desloratadine exhibited a significant loss of intracellular Mn specifically and a severe structural damage to the bacterial cell wall. Our results support the possibility of developing a novel, targeted therapy to treat Lyme disease by targeting specific metabolic needs of Borrelia.

Keywords: Lyme disease, BmtA, Borrelia burgdorferi, desloratadine, Bac Titer-Glo assay

\section{Introduction}

Since its establishment as a disease causing juvenile arthritis in the town of Lyme $^{1}$ and the discovery of Borrelia burgdorferi (B. burgdorferi) as the causative agent of this zoonosis, ${ }^{2,3}$ Lyme disease is turning out to be the single most prevalent vector-borne disease in the northern hemisphere. The Center for Disease Control and Prevention (CDC) recently updated the number of new Lyme disease patients from 30,000 to 300,000 per year in the United States of America. ${ }^{4,5}$ Whether this significant increase is due to excessive human invasion of natural habitats or improvements in diagnostic techniques, over the past two decades, is debatable. However, it is clear that the disease is prevalent and requires the urgent attention of the clinical and research community.

Early detection of Lyme disease and a full course treatment with tetracycline antibiotics leads to full recovery. ${ }^{6,7}$ However, in cases of late or misdiagnosis, a small fraction of the Lyme disease patients continue to exhibit neurological and arthritic symptoms associated with the disease, even after a full course of antibiotics. There are only few reports confirming the existence of posttreatment Lyme disease syndrome $(\mathrm{PTLDS})^{8-10}$ and the topic still remains somewhat debatable.

According to the $\mathrm{CDC}$ report, which was obtained by a series of clinical trials on the PTLDS, ${ }^{4,11-15}$ long-term antibiotic treatment for PTLDS patients is almost ineffective in 
suppression of PTLDS symptoms. Therefore, more in-depth research should be performed for accurate diagnosis and also for the development of effective therapeutics for PTLDS. During recent years, some groups have reported evidence of PTLDS in animal models ${ }^{16,17}$ which may strengthen the argument in favor of existence of this disease. It is reasonable to assume that current therapies may not be effective against PTLDS. Whether this is due to the persistence of infection or an autoimmune response to the infection is controversial. Nevertheless, if indeed this phenomenon is caused by persistent infection, elimination of bacteria from the system may be considered as the most suitable approach to resolve the problem. Thus, an alternative to currently prescribed antibiotics that is able to kill B. burgdorferi and help eliminate the infection in vivo is necessary.

Ouyang et $\mathrm{al}^{18}$ reported the presence of a metal transporter "BmtA (BB0219)" in B. burgdorferi. By induction of the efficient BmtA mutants, the authors found that intracellular accumulation of manganese $(\mathrm{Mn})$ was significantly decreased demonstrating that BmtA was responsible for the Mn uptake. Mn plays a crucial role in B. burgdorferi survival; for instance, $\mathrm{Mn}$ is a necessary cofactor for the B. burgdorferi superoxide dismutase machinery that protects the pathogen against intracellular superoxides. ${ }^{19}$ In addition, B. burgdorferi utilizes $\mathrm{Mn}$ as a cofactor for its various enzymes and protein functions ${ }^{18-23}$ instead of other commonly used metal ions such as iron. ${ }^{21}$ Therefore, one can expect that disruption of the Mn transporting systems across the $B$. burgdorferi cell membrane may lead to severe deficiencies in function and viability of B. burgdorferi.

Here, we report identification of novel compounds that can specifically bind to the predicted structure of B. burgdorferi metal transporter A (BmtA) and exhibit an inhibitory effect on B. burgdorferi growth. The efficacy of the identified compounds were determined by using novel Bac Titer-Glo microbial cell viability assay and direct cell counting under a light microscope. We further substantiate our hypothesis using a variety of techniques such as electron microscopy and inductively coupled plasma-mass spectrometry (ICP-MS) and discuss the possibility of utilizing BmtA inhibitory compounds as a new class of therapeutics to effectively treat Lyme disease either in combination or as an alternative to the currently available therapies.

\section{Materials and methods}

\section{Bacterial strains and culture}

B. burgdorferi sensu stricto strains CA4, CA8, wild type 297 (WT297), BmtA mutant strains OY04/04 and mockcomplemented OY07/F62 ${ }^{18}$ strains were cultured in BarbourStoner-Kelly II (BSK-II) complete medium, with 6\% rabbit serum (Sigma, St Louis, MO, USA). The procedure for construction of BmtA mutants was explained in Ouyang et al. ${ }^{18}$ The cultures were incubated at $33^{\circ} \mathrm{C}$ and maintained in sterile $50 \mathrm{~mL}$ falcon tubes. All culture media were sterilized with $0.2 \mu \mathrm{M}$ filter units (Millipore, Billerica, MA, USA). The B. burgdorferi cultures were grown for 7-10 days to reach the stationary phase for drug screening.

\section{In silico high-throughput screening}

The BmtA structure was predicted from its sequence using the I-Tasser program. ${ }^{24}$ The final model chosen was that with the highest $\mathrm{C}$-score (confidence score) or confidence score and prepared for Autodock Vina using Autodock tools. Specifically, charges and nonpolar hydrogens were added using default parameters in MGLTools. Autodock Vina 1.0.2 was used for all screenings in this study. The size of the docking grid was expanded from defaults to include the entire BmtA structure. The stringency of docking was set to 8 , the default parameter. Compounds used for screening were from the NCI Diversity Set II obtained from NCI and collections of FDA approved compounds obtained from www.drugbank.ca. The NCI Diversity Set II is a collection of around 2,000 compounds that are a part of the full NCI screening collection. Most of the compounds in this library are relatively rigid with five or fewer rotatable bonds allowing for a fast initial round of screening to obtain an initial idea of the types of chemical structures with high affinity for BmtA. In addition, most compounds possess pharmacologically desirable characteristics. They do not belong to class of polycyclic aromatic hydrocarbon, electrophilic, or organometallic molecules. Structural representations of the NCI Diversity in Set II were obtained from the NCI website and converted to a protein data bank (pdb) format. Individual pdb files were prepared for docking using the prepare_ligand.py scripts form MGLTools 1.5.4 using the largest nonbonded fragment present. Structural representations of the FDA approved small molecules were created using Chem3D Pro 12.0, converted into pdb formats, and prepared for docking using the same prepare_ligand.py scripts form MGLTools 1.5.4.

The free energies of binding in $\mathrm{kcal} / \mathrm{mol}$ as produced in Autodock Vina were used to score the ligand affinity with a lower free energy indicating greater affinity of the ligand for the protein.

\section{Determination of minimum inhibitory concentration (MIC) and minimum bactericidal concentration (MBC)}

The minimum inhibitory concentration (MIC) of the small molecules screened were determined by culturing $10^{6} / \mathrm{mL}$ 
Borrelia in BSK-II medium with different concentrations $(1-500 \mu \mathrm{M})$ of drugs. For MIC, the $1.2 \mathrm{~mL}$ cultures were grown in $1.5 \mathrm{~mL}$ microcentrifuge tubes in triplicates, wrapped with parafilm, and placed in the incubator for 72 hours at $33^{\circ} \mathrm{C}$. After the 3 -day incubation period, the microcentrifuge tubes were removed and observed for motile spirochetes in culture by using bacterial counting chamber (Petroff-Hausser Counter, Horsham, PA, USA).

For determining minimum bactericidal concentration (MBC), $20 \mu \mathrm{L}$ of the $10^{6} / \mathrm{mL}$ Borrelia cultures grown in BSK-II medium for 7 days at different drug concentrations were added to the fresh BSK-II medium and subcultured for 21 days. After 3 weeks (21 days) of incubation period, the samples were observed microscopically for motile spirochetes in culture. The MIC and MBC determinations were done thrice independently.

\section{Bac Titer-Glo microbial cell viability assay}

About $200 \mu \mathrm{L}$ of $10^{6} / \mathrm{mL}$ Borrelia strains were grown in white opaque bottom 96-well plates with drugs at respective concentrations ranging from $0 \mu \mathrm{M}$ to $250 \mu \mathrm{M}$. After 96 hours, $100 \mu \mathrm{L}$ of culture was taken from each well and mixed with $100 \mu \mathrm{L}$ of Bac Titer-Glo ${ }^{\circledR}$ reagent (Promega, Madison, WI, USA). Then, the assay was performed according to the manufacturer's instructions. Luminescence was measured on a Flex Station 3 micro plate reader at an integration time of 500 milliseconds.

\section{Formulation of small molecules}

The $100 \mathrm{mM}$ desloratadine (SantaCruz Biotech, SantaCruz, CA, USA) and loratadine (Ark Pharm, Libertyville, IL, USA) stock solutions were made by dissolving in $100 \%$ ethanol. $1 \mathrm{~mL}$ of $10 \mathrm{mM}$ working solutions was made by mixing $100 \mu \mathrm{L}$ of $100 \mathrm{mM}$ stock solutions in $250 \mu \mathrm{L} 20 \%$ hydroxypropyl $\beta$-cyclodextrin (Sigma) and $650 \mu \mathrm{L}$ of MilliQ water. The vehicle for cyclodextrin control was made by mixing $100 \mu \mathrm{L}$ of $100 \%$ ethanol in $250 \mu \mathrm{L} 20 \% \beta$-cyclodextrin and $650 \mu \mathrm{L}$ of MilliQ water. It is important to note that the vehicle control was identical to the test formulation in every single aspect except for the active ingredient. This measure was strictly followed for every vehicle control used in this assay. A stock solution of 10 $\mathrm{mM}$ yohimbine (Sigma) was prepared by dissolving in MilliQ water. A stock solution of $10 \mathrm{mg} / \mathrm{mL}$ of doxycycline was made by dissolving the doxycycline powder in MilliQ water.

\section{Analysis of intracellular metal contents by ICP-MS}

To determine the intracellular Mn metal contents, $10^{6} / \mathrm{mL}$ of CA4 strain was grown in BSK-II medium at $33^{\circ} \mathrm{C}$ for
7 days. Samples were washed twice each with phosphate buffered saline (PBS) and trace metal-free distilled water (Sigma). The pellets were dried in the oven at $95^{\circ} \mathrm{C}$ overnight, and dry weights of the pellets were recorded. The pellets were then lysed by using $65 \%$ trace metal-grade nitric acid (Sigma) and heated for 5 minutes at $85^{\circ} \mathrm{C}$. Finally, the supernatant was collected and diluted with trace metal-grade water (Sigma) to reach a final concentration of $2 \%$ nitric acid. Supernatants were filtered through a 0.22 $\mu \mathrm{M}$ filter (Millipore) and subjected to trace metal analysis by using inductively coupled plasma atomic emission spectrometry (ICP-Mass Spectrometer Thermo XSeries II) by the Environmental Measurements Facility (EM-1), Stanford University. Trace metal-grade standards were purchased from Sigma (Multielement Standard solution I for ICP from Sigma). The experiment was conducted twice independently.

\section{Transmission electron microscopy}

Cultures of B. burgdorferi were treated with $200 \mu \mathrm{M}$ desloratadine for 7 days in BSK-II medium. The cells were fixed with $4 \%$ glutaraldehyde in sodium cacodylate buffer $(0.1 \mathrm{M}$ [pH 7.2]). The samples were stained, dehydrated, and embedded in epoxy resin. Samples were sectioned into ultrathin sections of $100 \mathrm{~nm}$ with a glass knife and an ultramicrotome. Finally, sections were stained with 3.5\% uranyl acetate for 15 minutes and $0.2 \%$ lead citrate for 1 minute. Analysis of the sections was performed using a JEOL JEM 1400 transmission electron microscope (TEM) (JEOL USA, Inc., Peabody, MA, USA) at $120 \mathrm{kV}$ and digital images were taken with a GATAN Multiscan 701 digital camera (Western USA Sales, Pleasanton, CA, USA).

\section{Results}

\section{In silico folding of BmtA}

I-Tasser predicted four model structures for BmtA (Table 1) (gufA). The structure with the highest C-score was used as the final model. The pdb structures similar to the model structure of BmtA included importins (pdb id 1EE4 and 1PJM) and catenins (3L6X and 1I7W), Table 1. Ouyang et $a{ }^{18}$ revealed that $\mathrm{BB} 0219$ possesses the signature sequence (AxxxHNxxxGLAVG) unique to ZIP family zinc-regulated metal transporters (ZRTs) and iron-regulated metal transporters (IRTs)-like protein family, which are involved in uptake of $\mathrm{Zn}, \mathrm{Fe}$, or Mn. Though they are implicated by predicting signature sequence (AxxxHNxxxGLAVG) is in the conserved spanner IV and the central histidine involves in metal ion-binding site, from our structure prediction, at this point, we could not infer the means by which BmtA 
Table I List of proteins with similar structures to BmtA

\begin{tabular}{lll}
\hline pdb id & No of atoms overlaid & RMSD (A) \\
\hline IEE4 (importin) & 145 & 7.2 \\
2JDQ (importin) & 415 & 7.8 \\
3L6X (catenin) & 732 & 12.4 \\
II7W (catenin) & 602 & 9.8 \\
\hline
\end{tabular}

transports Mn. An inspection of the predicted BmtA structure reveals five alpha helices that could span a membrane. Charged groups (blue: positive and red: negative, Figure 1) are located at the edges of the helices, suggesting that most of the helices are hydrophobic and, consequently, could span the membranes.

\section{In silico screening process of compound libraries}

The NCI Diversity Set II compounds were screened against BmtA as described above by using Autodock Vina. Five percent of compounds with the highest binding affinities were selected (Figure 2A) and classified according to common structural groups. The FDA approved drug database was then visually inspected for molecules with similar structural groups as those with highest binding affinities. These molecules, desloratadine and yohimbine, were screened against BmtA (Figure 2B, Table 2). Both molecules possessed high binding affinities to BmtA and were, subsequently, evaluated in vitro.

\section{Analyzing effects of candidate molecules on $B$. burgdorferi survival in vitro} Assay development for B. burgdorferi survival

After establishing the in silico BmtA structure and using it for screening and shortlisting putative candidates, we optimized a novel in vitro biochemical assay for analyzing the effects of the predetermined compounds (eg, desloratadine

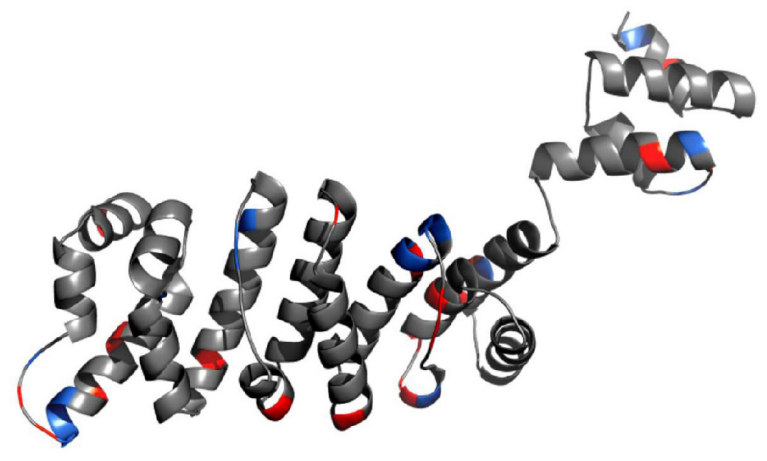

Figure I In silico folding of BmtA using the I-Tasser program. Notes: Positive and negatively charged groups are labeled blue and red, respectively. and yohimbine) on the B. burgdorferi survival. Various methods have been established to estimate bacterial survival and death. ${ }^{25}$ These methods can be broadly classified as direct visualization under the microscope, biochemical or enzymatic activity, and/or colorimetric assays. While each assay has certain advantages, they also suffer from severe limitations. For example, direct visualization of the bacteria under a microscope can yield direct and mostly unambiguous results, nonetheless, it is laborious and extremely low-throughput and thus has a limited potential to be used in compound screening. The conventional assays (eg, microdilution) can be effective for rapidly growing organisms that can significantly change medium color over a period of time but do suffer from a high rate of observer's error. Our goal was to establish a highly sensitive and automated assay with scale-up capability; which could also function over a few orders of magnitude and with negligible experimental bias. The Bac Titer-Glo reagent developed by Promega essentially measures adenosine $5^{\prime}$-triphosphate (ATP) content of the given culture. ATP, which is only being produced by living cells, can serve as an excellent marker for differentiating between live and dead cells. Bac Titer-Glo reagent is formulated by combining cell lysis buffer as well as luciferase in one solution to eliminate the need for in-between washes and has been successfully employed for studying viability of various types of bacteria. ${ }^{26}$ We have effectively used this assay to look at Borrelia cell viability. The signal has a good dynamic range and has been reported to detect signal from as few as ten bacteria, ${ }^{27}$ under optimal conditions. Since we were interested in studying the effects of drugs (eg, desloratadine and yohimbine) in physiologically relevant conditions, all candidate drug assays have been carried out in the BSK-II medium. The Bac Titer-Glo assay is very sensitive and can pick up even a minute ATP signal. $Z^{\prime}$ factor is used as a standard to determine sensitivity of the assay and whether the signal to noise ratio is significantly high to yield reliable results. Ideally, $Z^{\prime}$ value ranges between -1 and +1 and $Z^{\prime}$ value close to 1 is considered as "optimal" for such a sensitive assay. In white opaque 96-well plates, the assay could reliably detect cells at and above $7 \times 10^{3}$ in the BSK-II medium (Figure 3 ). The Bac Titer-Glo assay was validated by calculating $Z^{\prime}$ values for CA 8 and CA4 strains which are 0.7 and above, a good value to perform an HTS assay.

\section{Effects of candidate molecules on B. burgdorferi survival}

The WT297 and CA4 strains were employed for our assays. We have performed all our studies by using persisters 

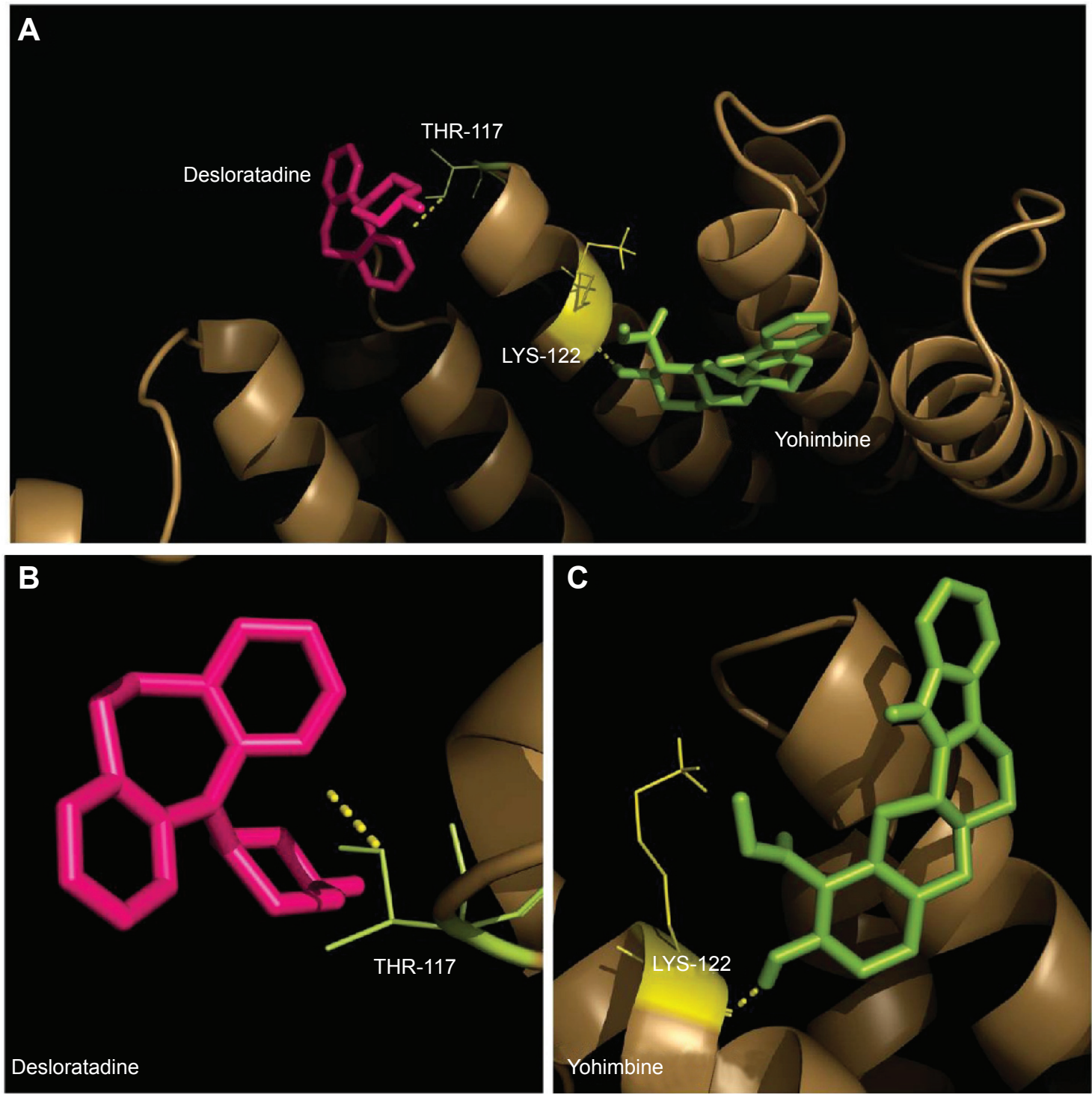

Figure 2 Predicted structure of BmtA using l-Tasser and predicted binding sites and amino acids involved in the interactions with desloratadine (magenta) and yohimbine (green) (A). A zoomed view of desloratadine interaction (B). A zoomed view of yohimbine interaction (C).

which are maintained for 7-10 days. The persisters have a stationary growth phase. The spirochetes were grown in BSK-II media and were kept at a low passage number to prevent loss of plasmids and virulence. For each assay $10^{6} / \mathrm{mL}$ spirochetes were used. The spirochetes were exposed to different concentrations of the drug for 96 hours and cell

Table 2 List of FDA approved candidate compounds exhibiting a strong binding to $\mathrm{BmtA}$

\begin{tabular}{ll}
\hline Molecule & Affinity $(\mathrm{kcal} / \mathrm{mol})$ \\
\hline Yohimbine & -8.2 \\
Loratadine & -8.0 \\
Desloratidine & -7.9 \\
3-hydroxydesloratidine & -8.1 \\
\hline
\end{tabular}

viability was measured using the Bac Titer-Glo reagent. Spirochetes in BSK-II medium without any treatment were used as a control in each experiment to determine normal growth of the spirochetes over time and were used to set $100 \%$ viability value in the survival curves. As expected, a dramatic decrease in cell viability was observed with even the lowest employed concentration of doxycycline in the assay. The obtained results were in good agreement with the published data. ${ }^{25}$ Cyclodextrin solution, which was used as a vehicle control (Figure 4) for desloratadine, did not show any significant effect on the cell survival. Desloratadine showed a potent inhibitory effect at and above $150 \mu \mathrm{M}$ ( $\sim 11 \%$ viability). Interestingly, yohimbine demonstrated a dramatic decrease in cell viability $(56 \%$ viability at $25 \mu \mathrm{M})$ 


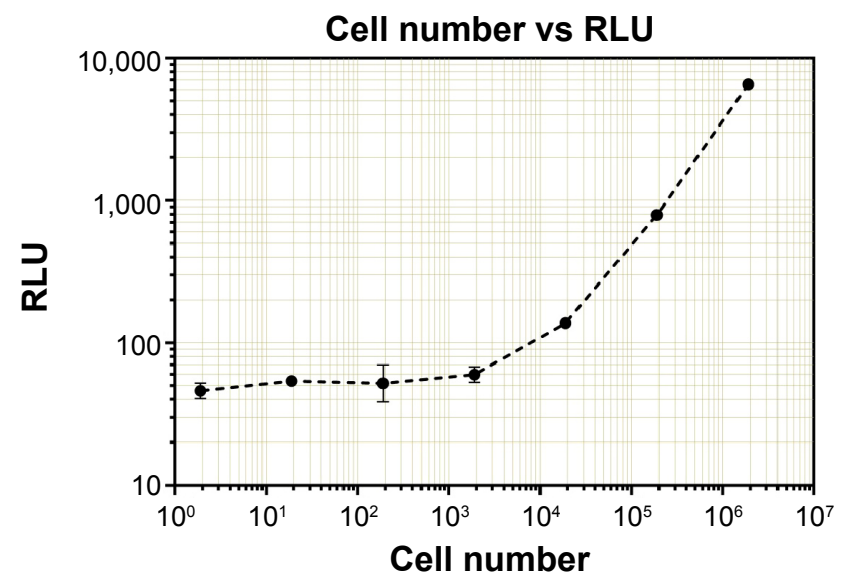

Figure 3 Optimization and sensitivity analysis of Bac Titer-Glo assay for screening the borreliacidal compounds.

Notes: Cell number vs luminescence. The results represent mean \pm SD. Abbreviations: SD, standard deviation; RLU, relative luminescence units.

(Figure S1). This effect, however, did not show concentration dependent increase above $25 \mu \mathrm{M}$, and even at the highest employed concentration $(250 \mu \mathrm{M})$ about $41 \%$ cell viability was observed (Figure 4). The data shown in Figure 4 is the results obtained from WT297 and CA4.

Exposure of the spirochetes to desloratadine and yohimbine exhibited formation of round bodies. While the Bac Titer-Glo assay can precisely predict the cell viability, it cannot discriminate the inhibitory or bactericidal effects of the drugs. In order to confirm that the drugs indeed have a lethal effect on spirochetes, we conducted an MBC/MIC assay by incubating $B$. burgdorferi from 72 hours up to 7 days in presence of drugs followed by inoculating the treated bugs in fresh medium for another 21 days. The growth was

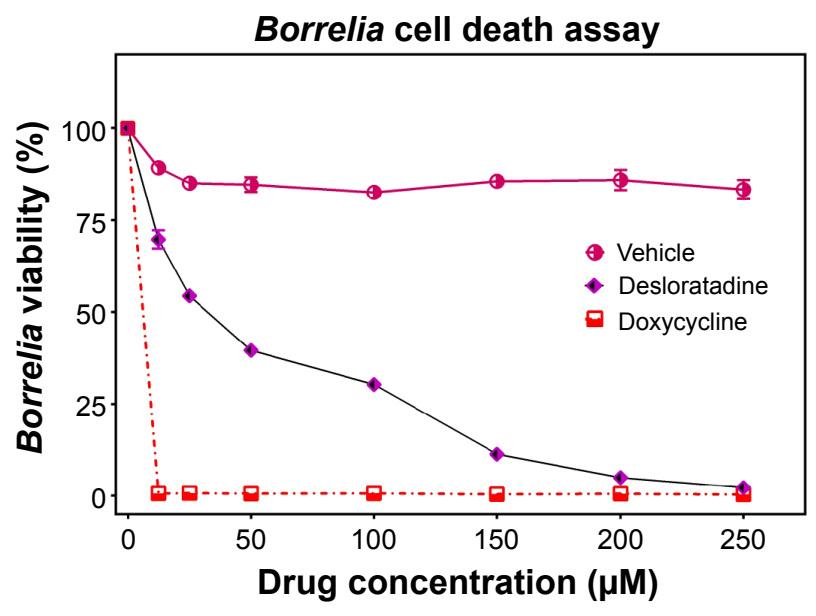

Figure 4 Effect of desloratadine on WT297 viability.

Notes: Doxycycline was used as a positive control while vehicle (cyclodextrin with ethanol) was used as a background control. The results represent mean $\pm S D$. Abbreviations: WT, wild type; SD, standard deviation. not observed at $250 \mu \mathrm{M}$ by direct cell counting under light microscope for desloratadine; we have determined MIC and $\mathrm{MBC}$ for desloratadine to be $250 \mu \mathrm{M}(77.7 \mu \mathrm{g} / \mathrm{mL})$. Yohimbine did not exhibit any bactericidal effect.

\section{Analysis of intracellular Mn content}

As BmtA was shown to be responsible for Mn transport, we hypothesized that compounds that show a strong binding to BmtA transporter may be able to decrease Mn uptake by the spirochetes, we now intended to check whether indeed the inhibitory effects exhibited by the compounds are due to decrease in the intracellular Mn levels. In this case we lysed bacterial pellets, which were obtained from drug treated or untreated samples, in $65 \%$ nitric acid and subjected the cellular extracts to ICP-MS analysis. Since the pellet size was considerably different, the data was normalized to the dry weight of the pellets. Interestingly, spirochetes treated with $250 \mu \mathrm{M}$ desloratadine exhibited very small to negligible pellet as compared to that from control untreated samples. In order to obtain quantifiable amounts of the pellet, we conducted the experiments with $200 \mu \mathrm{M}$ desloratadine treatment. We observed a considerable decrease in Mn content at $200 \mu \mathrm{M}$ compared to the wild type (3.854 parts per billion (ppb) as opposed to $8.33 \mathrm{ppb}$ from wild type) (Figure 5). This result supported our hypothesis that desloratadine can indeed affect Mn transport system which in turn affects the cell viability.

\section{Morphological effects}

In order to visualize morphological effects of desloratadine on B. burgdorferi, we analyzed desloratadine treated

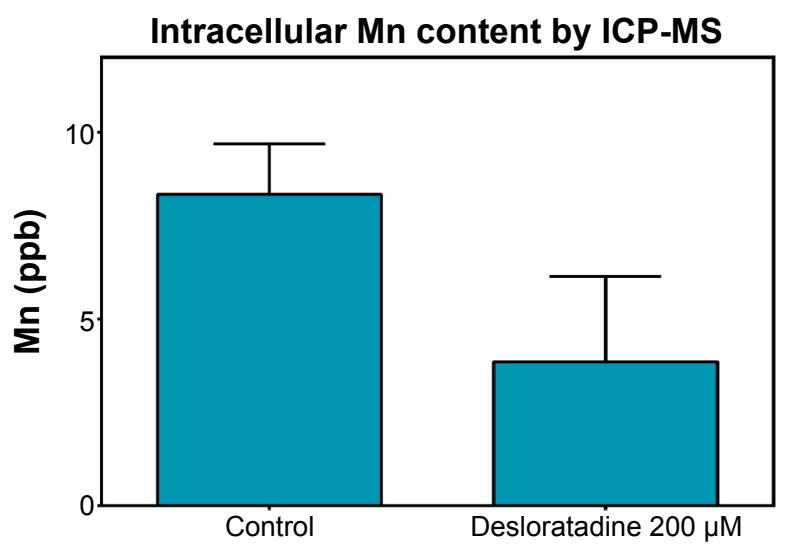

Figure 5 ICP-MS analysis of Mn content from desloratadine treated Borrelia and untreated control cells.

Notes: Values shown are ppb of Mn per mg of dry weight (DW). The results represent mean \pm SEM.

Abbreviations: ICP-MS, inductively coupled plasma-mass spectrometry; SEM, standard error of mean; ppb, parts per billion. 
spirochetes with immunofluorescence (IFA) and ultrastructure analysis by TEM. When the GFP labeled Borrelia were treated with $100 \mu \mathrm{M}$ desloratadine for 24 hours, numerous round bodies were observed (Figure S2). Based on the previous results studied with different drugs, ${ }^{18}$ we revealed that desloratadine treatment caused a massive round body formation and also a significant reduction in bacterial pellet size and mass. The findings strongly suggested a loss of structural integrity and destruction of the cell wall after drug treatment. TEM analysis showed the fact that indeed desloratadine treated spirochetes had massive structural deformities (see Figure 6). In desloratadine treated samples, numerous small, empty vacuoles were observed which appeared significantly smaller than typical round body structures reported by others. ${ }^{28}$ The data strongly suggest that desloratadine can cause irreversible damage to B. burgdorferi, possibly by blocking Mn transporting system that ultimately results in cellular disintegration.

\section{Effects of desloratadine on BmtA mechanism as well as bacterial growth in BmtA-deficient mutants}

To determine whether the effect caused by desloratadine on Borrelia is due to targeting Mn transport of BmtA gene, efficacy of desloratadine was studied on $B m t A$-deficient mutants OY07/F62, OY04/04, and WT297. The WT297 and $B m t A$-deficient mutants were grown in BSK-II medium with respective concentration of desloratadine mentioned. The desloratadine effect on WT297, OY07/F62, and OY04/04 is shown in Figure 7 (A, B, C), respectively. In both the BmtAdeficient mutants the viability is more than the WT297. The viability of the WT and BmtA-deficient mutants are depicted in Figure 7D and analyzed as follows. For OY04/04 mutant the viability is higher than WT297 by $48 \%, 61 \%, 72 \%$, $68 \%, 53 \%, 30 \%$, and $19 \%$ in desloratadine treated cultures at concentrations $12.5,25,50,100,150,200$, and $250 \mu \mathrm{M}$, respectively. At desloratadine concentrations 12.5, 25, 50, $100,150,200$, and $250 \mu \mathrm{M}$, the viability is more by $18 \%$, $55 \%, 72 \%, 68 \%, 53 \%, 30 \%$, and $11 \%$, respectively, in OY $07 /$ F62 mutant, when compared to the WT297. In WT297 viability is significantly decreased with increasing concentrations of desloratadine when compared to mutants OY04/04 and OY07/F62. The viability of mutants OY04/04 and OY07/ F62 is significantly more or nearly same as the vehicle till $100 \mu \mathrm{M}$ concentration. At concentrations of 200 and $250 \mu \mathrm{M}$, though, the viability of mutants is very low compared to the vehicle, and the overall viability of the BmtA mutants is higher compared to the WT297.

\section{Discussion}

Metal homeostasis is strongly controlled within the cells due to the critical roles it plays in bacterial virulence, gene regulation, defense against ROS, and metabolism. . $82,22,29^{\text {Different }}$ microbes have elegant metal transporters regulating the metal homeostasis..$^{30-33}$ Hence, it is reasonable to hypothesize that disturbance in metal transition across cell membrane by pharmacological interference can be a promising approach to kill/ inhibit B. burgdorferi. Different bacteria depend on different metal ions such as iron, manganese, and zinc for their survival. Recently, it has been suggested that the therapeutic strategies to destroy or inhibit the microbes are highly specific to respective microbial strains..$^{34,35}$ It means that various microbes respond differently to therapeutic agents owing to their particular properties. It can be concluded that comprehensive knowledge about the susceptibility, adaptation capability, cycles, growth, resistance mechanism, vital biomolecules, and process
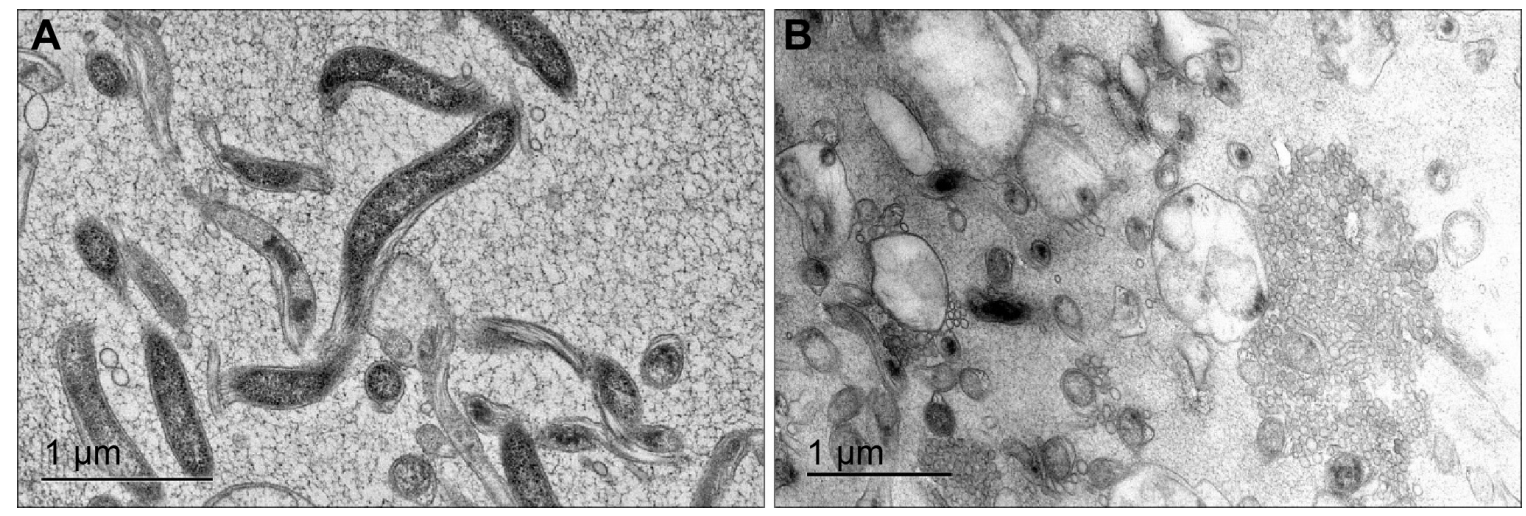

Figure 6 Ultrastructural analysis of untreated (A) and desloratadine treated (B) Borrelia burgdorferi by TEM.

Notes: B. burgdorferi CA4 was treated with $200 \mu \mathrm{M}$ of desloratadine and cultured for 7 days in BSK-II medium. (TEM; scale bar: I $\mu \mathrm{m}$ ).

Abbreviations: BSK-II, Barbour-Stoner-Kelly II; TEM, transmission electron microscopy. 
A

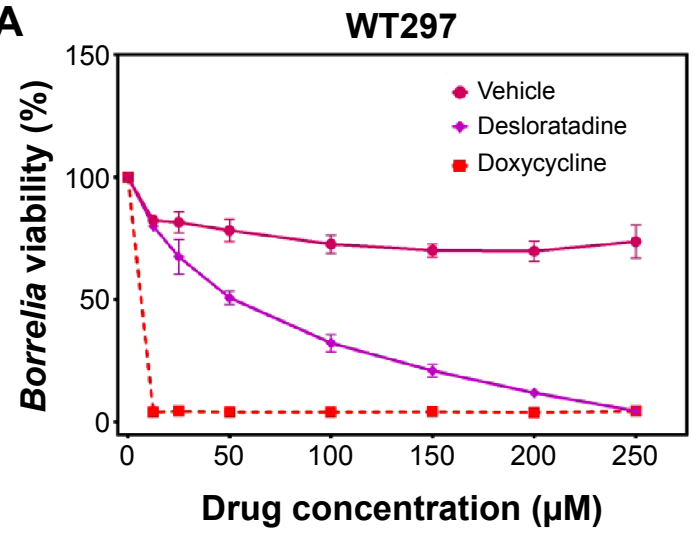

C

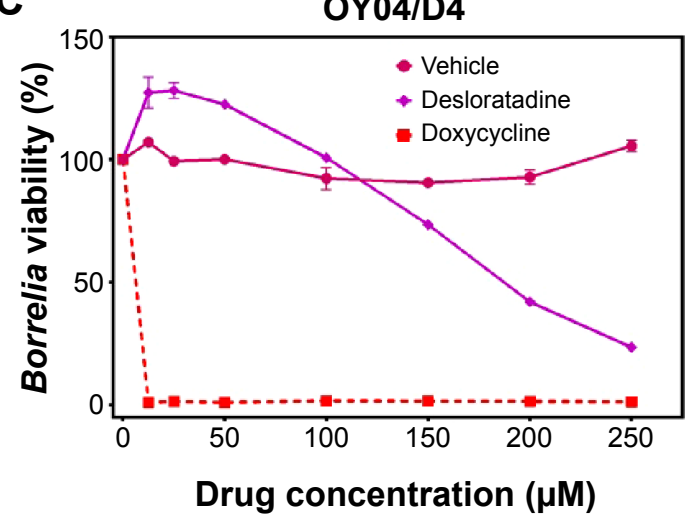

B

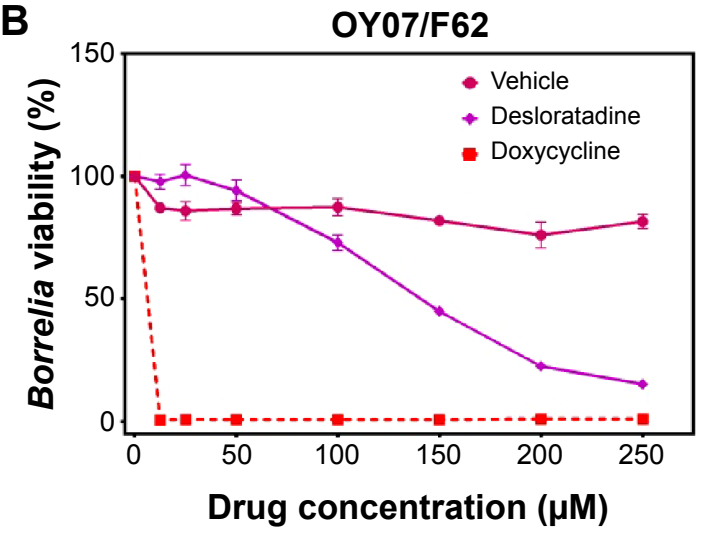

BmtA mutant

D

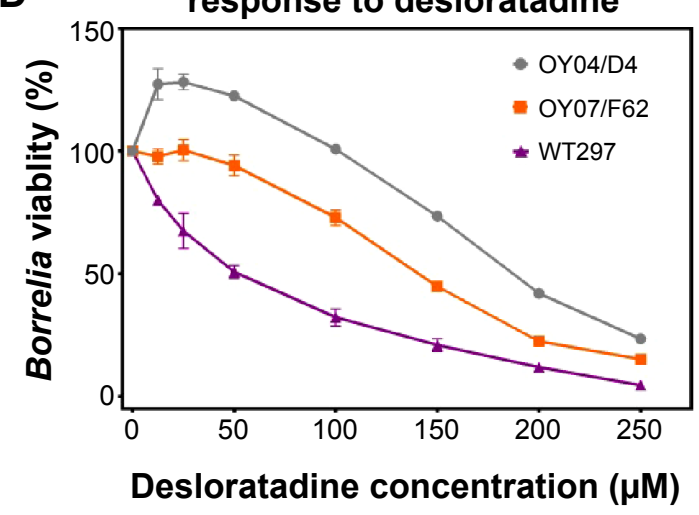

Figure 7 Effect of desloratadine on BmtA-deficient mutants (A) WT297, (B) mock-complemented OY07/F62, and (C) OY04/04.

Notes: Doxycycline was used as a positive control while vehicle was used as a control (D) BmtA mutants OY04/04, OY07/F62, and WT297 response to desloratadine. The results represent mean \pm SD.

Abbreviations: WT, wild type; SD, standard deviation.

of microbes may be helpful to choose the best strategy with maximal therapeutic efficacy and minimal side effects.

For B. burgdorferi, iron was shown to play a negligible role in cell viability. ${ }^{21}$ It was revealed that $\mathrm{Mn}$ rather than iron plays a critical role in B. burgdorferi life cycle mainly through BmtA transporting mechanism. The ultimate goal of this study is identification of compounds that could interfere with BmtA function and effectively block Mn transport across Borrelial cell wall in vitro. This study is also aimed at developing a targeted drug discovery program that particularly looks for agents that could specifically block transport of essential metabolites and nutrients across the cell wall. This study provides an assay platform to identify such compounds and characterize their effects on Borrelia by different assays. In order to reach this goal, we have first employed an in silico high-throughput screening approach to find suitable formulations to target the $\mathrm{Mn}$ transporter in B. burgdorferi. According to the obtained in silico results, several FDA approved compounds with high affinity and selectivity to BmtA transporter were shortlisted. We then optimized in vitro assays (eg, ATP-based cell viability assay, microdilution assay, and bacterial counting) to determine efficacy of these compounds to 1) eliminate Borrelia and 2) specifically reduce intracellular Mn concentration (eg, ICP-MS). The data obtained from these studies strongly indicated that desloratadine is able to kill Borrelia in vitro possibly by blocking Mn transport in Borrelia. We however must acknowledge that this study has certain ambiguities.

Ideally, a demonstration of direct binding between BmtA and desloratadine by any of the standard biophysical techniques such as cocrystallization of the two molecules, isothermal titration calorimetry (ITC), or surface plasmon resonance (SPR) would be the most critical experiment to substantiate this claim. BmtA is a challenging protein to work with since a lot of information about its structure is still missing. It is a seven transmembrane domain protein and that adds further challenges to its expression and purification in another bacterial expression system such as Escherichia coli. We were able to clone BmtA from Borrelia and optimize the expression of recombinant BmtA in E. coli for biophysical 
studies (data not shown). The studies however, were not pursued further due to very poor yield of the protein (17 $\mu \mathrm{g} / \mathrm{L})$ in initial experiments.

Desloratadine is a promising safe drug (approved by FDA as allergy controller ${ }^{36,37}$ ) that is able to inhibit/kill B. burgdorferi. Mn plays a critical role in Borrelia survival and growth; however, elimination of BmtA transporter does not kill the bacterium. Since desloratadine treatment not only inhibits BmtA but also kills the bacteria and results in severe structural damage, one is compelled to speculate the existence of at least one additional mechanism by which desloratadine may be able to cause lethal effects. There is some experimental evidence that suggests that antihistaminic compounds could damage bacterial cell wall. ${ }^{38}$ Even though this has not been tested or reported for desloratadine, it could be a possibility based on our results. The lack of increase in activity for yohimbine above $25 \mu \mathrm{M}$ suggested a limited availability of this molecule after a critical concentration. Since yohimbine did not exhibit a significant effect on Borrelia survival, we did not further study if yohimbine actually affects Mn transport experimentally. Factors such as aggregation at increased concentration may contribute to limited availability of the free drug. Tadalafil, even though predicted to be a molecule exhibiting similar binding and possibly similar effect, could not be used in the assay due to technical challenges associated with its formulation compatible with culture medium. Desloratadine could change the intracellular Mn homeostasis and its transition across cell membrane. Our results showed that desloratadine treatment and subsequent decrease in the intracellular Mn concentration play an important role in Borrelia structure integrity and viability.

Boylan et $\mathrm{al}^{39}$ demonstrated that since $B$. burgdorferi has no intracellular iron, the reactive oxygen species (ROS) mainly targeted membrane lipids but not DNA. As B. burgdorferi requires $\mathrm{Mn}$ in order to degrade the ROS, it is possible that blocking of Mn transition across cell wall may result in increasing of ROS level which in turn leads to disruption of cell membrane. To determine that the effect of desloratadine on Borrelia viability is due to Mn transport inhibition by BmtA gene, efficacy studies were done with BmtA-deficient mutants OY04/04, OY07/F62 (mock-complemented strain), and WT297. For both mutants when treated with desloratadine the Borrelia viability is higher in all concentrations compared to the WT297. But in mutant OY04/04 the viability is significantly higher than the OY07/F62 (mock-complemented strain). Both OY07/F62 and OY04/04 exhibited significantly higher growth with drug treatment (from 0 to $50 \mu \mathrm{M}$ ) than that of treatment with equivalent amount of vehicle. This shows that deletion of BmtA (bb0219) gene significantly increases the growth and protects Borrelia from desloratadine. Though the binding of desloratadine to BmtA is not studied, the experiment indirectly shows that desloratadine may bind to BmtA and inhibit the Mn transport.

As BmtA and Mn are dispensable when B. burgdorferi is cultivated in vitro; this might be one of the reasons that desloratadine has higher MIC and MBC values. It could be explained due to the fact that BSK medium does not recapitulate the physiological or metal conditions that B. burgdorferi encounters in either ticks or mammals. Based on these studies, it might be possible that desloratadine may show better efficacy in animal models than in vitro studies. So, animal studies in the near future may answer this question. Desloratadine exhibited potent borreliacidal activity in vitro at and above $78 \mu \mathrm{g} / \mathrm{mL}(250 \mu \mathrm{M})$. Currently, desloratadine is prescribed at 5-10 mg dose. A $250 \mu \mathrm{M}$ dose would roughly translate into $400 \mathrm{mg} /$ day. The mean plasma concentration of desloratadine in human blood after $5 \mathrm{mg}$ or even up to $40 \mathrm{mg}$ dose is about $2-5 \mathrm{ng} / \mathrm{mL} .{ }^{40}$ Thus, achieving borreliacidal concentrations of desloratadine in blood are challenging and $250 \mu \mathrm{M}$ concentration of desloratadine in vivo is physiologically irrelevant. However, this study provides compelling evidence that small molecules such as desloratadine could kill Borrelia. Efficacy of desloratadine-like molecules can be increased by designing modified derivatives of desloratadine that exhibit the same or better effect at considerably lower concentrations. The Bac Titer-Glo assay optimized by our group can be further employed to screen libraries of desloratadine derivatives. Achieving this could help to reduce the use of longer antibiotic treatment which leads to obvious side effects during treatment in Lyme patients.

In addition to studying the above proposed mechanism we also developed novel Bac Titer-Glo assay for screening the small molecules. The assay which was developed in 96-well format has a good $Z^{\prime}$ value of 0.7 and above. The Bac TiterGlo assay has many advantages compared to the other fluorescence methods developed for high-throughput screening of small molecules against $B$. burgdorferi. It is a single step assay and is less time consuming which is easy to adopt in a larger 384-well platform and screening of a large number of compounds. ${ }^{41,42}$ We also adopted this assay to 384-well format, and screening the FDA approved compounds in large scale is in progress. As every assay has its own limitations, the Bac Titer-Glo assay detection limit is little bit higher.

In summary, desloratadine treatment of B. burgdorferi can result in blocking of the $\mathrm{Mn}$ transporter BmtA of the bacterial cell wall and, consequently, has the potential to 
eliminate the B. burgdorferi infection. It can be considered as a new, nonantibiotic, antiborrelial compound for treating Lyme disease if the efficacy of the drug is improved by developing designer molecules. Whether Borrelia has the ability to develop resistance to desloratadine-like compounds is an important question and should be addressed in future studies.

\section{Acknowledgments}

This work was accomplished with a generous support from the Bay Area Lyme Foundation. We are indebted to Dr Robert Lane (University of California, Berkeley, CA) for his constant support, discussion, and provision of valuable bacterial strains for the study. We also thank Dr George Chaconas (University of Calgary, Canada) for providing the GFP-Borrelia strain for our studies. We are thankful to Michael V Norgard for generously sharing the BmtA mutants and WT297. We thank Dr Eliza Ruben, Dr Seongsoo Lee, Chris Wagstrom, Dr SiWan Kim, and other BioADD members for their support in this study. We also thank the Electron Microscopy Core and the Cell Science Imaging Facility (CSIF) at Stanford University for their support for ultra-structural studies.

\section{Disclosure}

Venkata Raveendra Pothineni, Dhananjay Wagh and Jayakumar Rajadas are listed on the following patent assigned to Stanford University: Inhibitors of Borrelia Metal Transporter for Treatment of Lyme Disease. All the authors report no conflicts of interest in this work.

\section{References}

1. Steere AC, Malawista SE, Snydman DR, et al. Lyme arthritis: an epidemic of oligoarticular arthritis in children and adults in three connecticut communities. Arthritis Rheum. 1977;20(1):7-17.

2. Burgdorfer W, Barbour AG, Hayes SF, Benach JL, Grunwaldt E, Davis JP. Lyme disease-a tick-borne spirochetosis? Science. 1982; 216(4552):1317-1319.

3. Steere AC, Grodzicki RL, Kornblatt AN, et al. The spirochetal etiology of Lyme disease. N Engl J Med. 1983;308(13):733-740.

4. Johnson L, Wilcox S, Mankoff J, Stricker RB. Severity of chronic Lyme disease compared to other chronic conditions: a quality of life survey. Peer J. 2014;2:e322

5. Kuehn BM. CDC estimates 300,000 US cases of Lyme disease annually. JAMA. 2013;310(11):1110.

6. Strickland GT, Caisley I, Woubeshet M, Israel E. Antibiotic therapy for Lyme disease in Maryland. Public Health Rep. 1994;109(6): 745-749.

7. Taylor RS, Simpson IN. Review of treatment options for lyme borreliosis. J Chemother. 2005;17(Suppl 2):3-16.

8. Aucott JN, Crowder LA, Kortte KB. Development of a foundation for a case definition of post-treatment Lyme disease syndrome. Int $J$ Infect Dis. 2013;17(6):e443-e449.

9. Aucott JN, Rebman AW, Crowder LA, Kortte KB. Post-treatment Lyme disease syndrome symptomatology and the impact on life functioning: is there something here? Qual Life Res. 2013;22(1):75-84
10. Aucott JN, Seifter A, Rebman AW. Probable late lyme disease: a variant manifestation of untreated Borrelia burgdorferi infection. BMC Infect Dis. 2012;12:173.

11. Cameron DJ. Insufficient evidence to deny antibiotic treatment to chronic Lyme disease patients. Med Hypotheses. 2009;72(6):688-691.

12. Delong AK, Blossom B, Maloney EL, Phillips SE. Antibiotic retreatment of Lyme disease in patients with persistent symptoms: a biostatistical review of randomized, placebo-controlled, clinical trials. Contemp Clin Trials. 2012;33(6):1132-1142.

13. Klempner MS. Controlled trials of antibiotic treatment in patients with post-treatment chronic Lyme disease. Vector Borne Zoonotic Dis. 2002;2(4):255-263.

14. Klempner MS, Baker PJ, Shapiro ED, et al. Treatment trials for postLyme disease symptoms revisited. Am J Med. 2013;126(8):665-669.

15. Klempner MS, Hu LT, Evans J, et al. Two controlled trials of antibiotic treatment in patients with persistent symptoms and a history of Lyme disease. N Engl J Med. 2001;345(2):85-92.

16. Bockenstedt LK, Gonzalez DG, Haberman AM, Belperron AA. Spirochete antigens persist near cartilage after murine Lyme borreliosis therapy. J Clin Invest. 2012;122(7):2652-2660.

17. Embers ME, Barthold SW, Borda JT, et al. Persistence of Borrelia burgdorferi in rhesus macaques following antibiotic treatment of disseminated infection. PLoS One. 2012;7(1):e29914.

18. Ouyang Z, He M, Oman T, Yang XF, Norgard MV. A manganese transporter, BB0219 (BmtA), is required for virulence by the Lyme disease spirochete, Borrelia burgdorferi. Proc Natl Acad Sci U S A. 2009;106(9):3449-3454.

19. Troxell B, Xu H, Yang XF. Borrelia burgdorferi, a pathogen that lacks iron, encodes manganese-dependent superoxide dismutase essential for resistance to streptonigrin. J Biol Chem. 2012;287(23):19284-19293.

20. Aguirre JD, Clark HM, McIlvin M, et al. A manganese-rich environment supports superoxide dismutase activity in a Lyme disease pathogen, Borrelia burgdorferi. J Biol Chem. 2013;288(12):8468-8478.

21. Posey JE, Gherardini FC. Lack of a role for iron in the Lyme disease pathogen. Science. 2000;288(5471):1651-1653.

22. Troxell B, Yang XF. Metal-dependent gene regulation in the causative agent of Lyme disease. Front Cell Infect Microbiol. 2013;3:79.

23. Troxell B, Ye M, Yang Y, Carrasco SE, Lou Y, Yang XF. Manganese and zinc regulate virulence determinants in Borrelia burgdorferi. Infect Immun. 2013;81(8):2743-2752.

24. Roy A, Kucukural A, Zhang Y. I-TASSER: a unified platform for automated protein structure and function prediction. Nat Protoc. 2010; 5(4):725-738

25. Sapi E, Kaur N, Anyanwu S, et al. Evaluation of in-vitro antibiotic susceptibility of different morphological forms of Borrelia burgdorferi. Infect Drug Resist. 2011;4:97-113.

26. Junker LM, Clardy J. High-throughput screens for small-molecule inhibitors of Pseudomonas aeruginosa biofilm development. Antimicrob Agents Chemother. 2007;51(10):3582-3590.

27. Promega Corporation. Bac Titer-Glo microbial cell viability assay protocol. Promega; 2012.

28. Brorson O, Brorson SH, Scythes J, MacAllister J, Wier A, Margulis L. Destruction of spirochete Borrelia burgdorferi round-body propagules (RBs) by the antibiotic tigecycline. Proc Natl Acad Sci U S A. 2009; 106(44):18656-18661.

29. Véscovi EG, Soncini FC, Groisman EA. $\mathrm{Mg}^{2+}$ as an extracellular signal: environmental regulation of Salmonella virulence. Cell. 1996; 84(1):165-174.

30. Papp-Wallace KM, Maguire ME. Manganese transport and the role of manganese in virulence. Annu Rev Microbiol. 2006;60:187-209.

31. Wandersman C, Delepelaire P. Bacterial iron sources: from siderophores to hemophores. Аппи Rev Microbiol. 2004;58:611-647.

32. Ouyang Z, Isaacson R. Identification and characterization of a novel $\mathrm{ABC}$ iron transport system, fit, in Escherichia coli. Infect Immun. 2006;74(12):6949-6956.

33. Patzer SI, Hantke K. Dual repression by $\mathrm{Fe}^{2+}-\mathrm{Fur}$ and $\mathrm{Mn}^{2+}$-MntR of the mntH gene, encoding an NRAMP-like $\mathrm{Mn}^{2+}$ transporter in Escherichia coli. J Bacteriol. 2001;183(16):4806-4813. 
34. Hajipour MJ, Fromm KM, Ashkarran AA, et al. Antibacterial properties of nanoparticles. Trends Biotechnol. 2012;30(10):499-511.

35. Ashkarran AA, Ghavami M, Aghaverdi H, Stroeve P, Mahmoudi M. Bacterial effects and protein corona evaluations: crucial ignored factors in the prediction of bio-efficacy of various forms of silver nanoparticles. Chem Res Toxicol. 2012;25(6):1231-1242.

36. Geha RS, Meltzer EO. Desloratadine: a new, nonsedating, oral antihistamine. J Allergy Clin Immunol. 2001;107(4):751-762.

37. Baena-Cagnani CE, Berger WE, DuBuske LM, et al. Comparative effects of desloratadine versus montelukast on asthma symptoms and use of $\beta 2$-agonists in patients with seasonal allergic rhinitis and asthma. Int Arch Allergy Immunol. 2003;130(4):307-313.

38. El-Nakeeb MA, Abou-Shleib HM, Khalil AM, Omar HG, E1Halfawy OM. Membrane Permeability Alteration of Some Bacterial Clinical Isolates by Selected Antihistaminics. Braz J Microbiol. 2011; 42(3):992-1000.
39. Boylan JA, Lawrence KA, Downey JS, Gherardini FC. Borrelia burgdorferi membranes are the primary targets of reactive oxygen species. Mol Microbiol. 2008;68(3):786-799.

40. Xu HR, Li XN, Chen WL, Chu NN. Simultaneous determination of desloratadine and its active metabolite 3-hydroxydesloratadine in human plasma by LC/MS/MS and its application to pharmacokinetics and bioequivalence. J Pharm Biomed Anal. 2007;45(4):659-666.

41. Feng J, Wang $\mathrm{T}$, Shi $\mathrm{W}$, et al. Identification of novel activity against Borrelia burgdorferi persisters using an FDA approved drug library. Emerg Microbes Infect. 2014;3(7):e49.

42. Cornell KA, Primus S, Martinez JA, Parveen N. Assessment of methylthioadenosine/S-adenosylhomocysteine nucleosidases of Borrelia burgdorferi as targets for novel antimicrobials using a novel high-throughput method. J Antimicrob Chemother. 2009;63(6): $1163-1172$. 


\section{Supplementary materials}

\section{B. burgdorferi cell death assay}

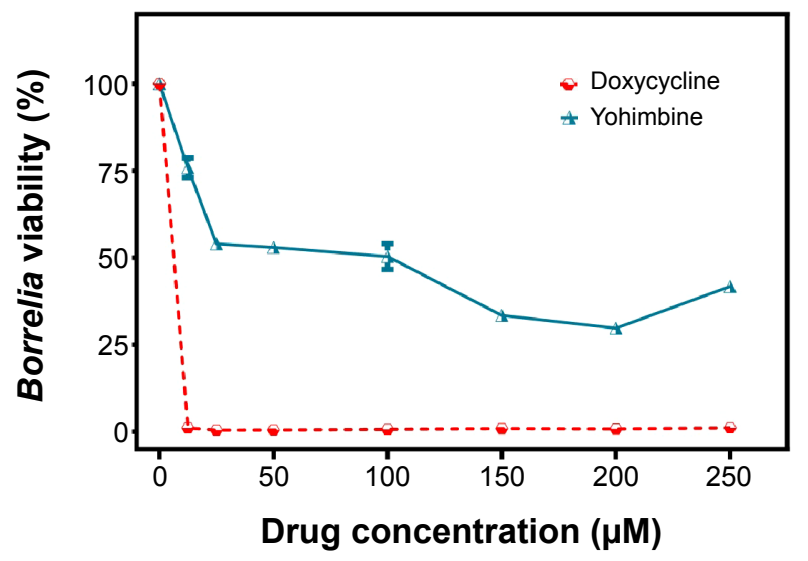

Figure SI Effect of yohambine on WT297 viability.

Notes: Doxycycline was used as a positive control. The results represent mean $\pm S D$.

Abbreviations: WT, wild type; SD, standard deviation.

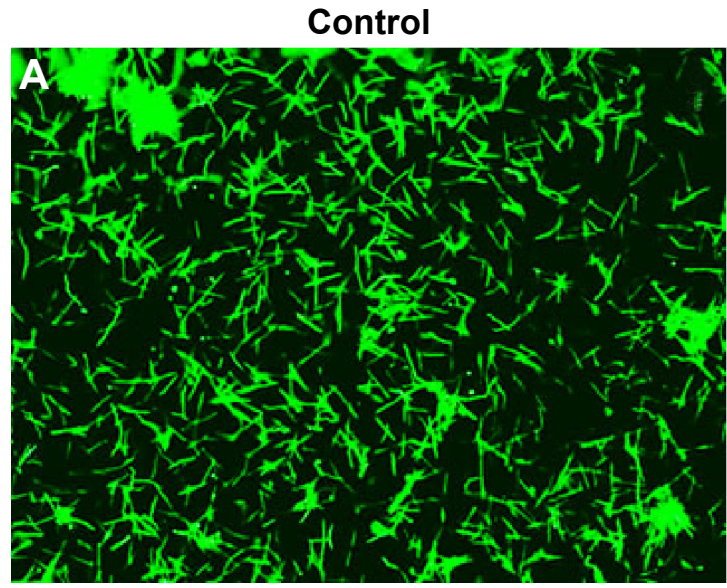

24 hours

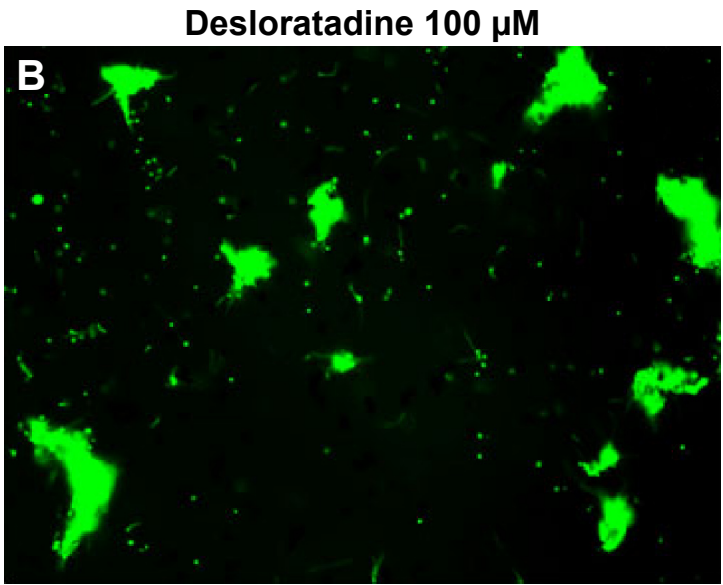

24 hours

Figure S2 Microscopic analysis of untreated (A) and desloratadine treated (B) Borrelia burgdorferi by IFA.

Notes: GFP labeled Borrelia was treated with $100 \mu \mathrm{M}$ of desloratadine and cultured for 24 hours in BSK-II medium.

Abbreviations: IFA, immunofluorescence; BSK-II, Barbour-Stoner-Kelly II.

\section{Publish your work in this journal}

Drug Design, Development and Therapy is an international, peerreviewed open-access journal that spans the spectrum of drug design and development through to clinical applications. Clinical outcomes, patient safety, and programs for the development and effective, safe, and sustained use of medicines are a feature of the journal, which has also been accepted for indexing on PubMed Central. The manuscript management system is completely online and includes a very quick and fair peer-review system, which is all easy to use. Visit http://www.dovepress.com/testimonials.php to read real quotes from published authors. 